\title{
Comparison of the American Thyroid Association with the Endocrine Society practice guidelines for the screening and treatment of hypothyroidism during pregnancy
}

\author{
Atieh Amouzegar, ${ }^{1}$ Ladan Mehran, ${ }^{1}$ Farzaneh Sarvghadi, ${ }^{1}$ Hossein Delshad, ${ }^{2}$ \\ Fereidoun Azizi, ${ }^{1}$ John H. Lazarus ${ }^{3}$
}

${ }^{1}$ Endocrine Research Center, ${ }^{2}$ Obesity Research Center; Research Institute for Endocrine Sciences, Shahid Beheshti University of Medical Sciences; Tehran, Iran; ${ }^{3}$ Centre for Endocrine and Diabetes Sciences, University Hospital of Wales, Cardiff School of Medicine; Wales, UK

\section{INTRODUCTION}

Thyroid hormones play a critical role in neurodevelopment both during pregnancy and postnatally. The fetal thyroid produces sufficient amounts of thyroid hormones just after 16 weeks of pregnancy prior to which the fetus is totally dependent on maternal FT4 for its physical and neural development. ${ }^{1,2}$ Estimation of the prevalence of thyroid diseases has been based on region, assay, and trimester-specific TSH cut-offs used for the diagnosis and amount of iodine intake during pregnancy. Carrying out a thyroid function survey using a general health checkup system for pregnant women actually increases the prevalence of thyroid dysfunction during this period. Overall subclinical hypothyroidism $(\mathrm{SCH})$ is reported to have a prevalence

Key words: Guideline, Hypothyroidism, Management, Pregnancy, Screening

\section{Address for correspondence:}

Fereidoun Azizi, Director Obesity Research Center Research Institute for Endocrine Sciences, Shahid Beheshti University of Medical Sciences, Tehran, I.R. Iran;

P.O. Box: 19395-4763, Tehran, I.R. Iran,

Tel.: + 982122432503 , Fax: + 982122402463 ,

E-mail: azizi@endocrine.ac.ir

Received 02-07-2013, Accepted 03-10-2014 of $1-2 \%$ of all pregnancies, ${ }^{3}$ with the prevalence of hypothyroidism being even higher in some studies. ${ }^{4}$ Using recent Endosociety guidelines for definition of subclinical hypothyroidism, i.e. a serum TSH $>2.5$ $\mathrm{mIU} / \mathrm{L}$ during the first and $\mathrm{TSH}>3 \mathrm{mIU} / \mathrm{L}$ during second and third trimesters, will also increase the prevalence of subclinical hypothyroidism. In a study, Shields et al reported the prevalence of subclinical hypothyroidism in pregnancy ( $\mathrm{TSH}>3 \mathrm{mIU} / \mathrm{L}$ ) to be $12.4 \%$ in pregnant women during routine antenatal care. ${ }^{5}$ Meanwhile, two recent reports have cited the prevalence of subclinical or clinical hypothyroidism as being $12.3 \%$ in a Finnish study, ${ }^{6}$ and $35.3 \%$ in a South American study. ${ }^{7}$ In order to determine presence of thyroid diseases, the trimester-specific reference range must be applied, current data showing the upper reference range to be $2.5 \mathrm{mIU} / \mathrm{L}$ during the first and $3 \mathrm{mIU} / \mathrm{L}$ during the second and third trimesters. ${ }^{8}$ Subclinical hypothyroidism $(\mathrm{SCH})$ is defined as TSH levels above 2.5 but less than $10 \mathrm{mIU} / \mathrm{L}$ in the presence of normal FT4 levels. Overt hypothyroidism is defined as serum TSH level $>2.5 \mathrm{mIU} / \mathrm{L}$ in conjunction with FT4 concentration at $<10^{\text {th }}$ percentile of the reference range or serum $\mathrm{TSH}$ level $\geq 10 \mathrm{mIU} / \mathrm{L}$, irrespective of FT4 levels. ${ }^{9}$ Overt maternal thyroid disease during pregnancy might not only seriously affect the somatic growth and neurocognitive ability of the fetus but could also cause adverse maternal out- 
comes such as placental abruption, preterm delivery or miscarriage. ${ }^{10-12}$ Reporting on hypothyroidism during pregnancy, Haddow et al have shown that mental performance of children born to a mother with even mild hypothyroidism can be adversely affected. ${ }^{10}$ In this study, treatment of hypothyroidism caused an increase in IQ scores in the children of women with serum thyrotropin concentrations at or above the 98th percentile. Before 16 weeks of pregnancy, when the fetus is totally dependent on maternal thyroid function for brain development, maternal thyroid sufficiency plays a vital role. Since neuronal migration and organization occurs subsequent to this point, thyroid insufficiency beyond 16 weeks is also harmful for the developing brain and has adverse consequences that will be responsible for delayed neuropsychological functions. It is still a matter of debate whether subclinical hypothyroidism could cause any serious adverse maternal or fetal events or result in lower IQ scores of children born to subclinically hypothyroid mothers and whether the treatment of such cases could result in any beneficial effects or prevent the development of adverse pregnancy outcomes. ${ }^{13-17}$ For example, the results of recent clinical trials by Lazarus et al showed that treatment of maternal subclinical hypothyroidism did not improve cognitive function in 3-year old children. ${ }^{18}$ Meanwhile, available data suggest that maternal hypothyroxinemia in the first half of pregnancy, but not later on, impairs cognitive development in infancy and childhood.

To answer the question as to who should be screened, a choice needs to be made between generalized screenings versus case-findings. What are the truly beneficial effects of treatment for pregnant women suffering from subclinical hypothyroidism and for their offspring? There is also a lack of strong data on the necessity of testing thyroid antibodies during pregnancy and how to manage those with positive thyroid antibodies. Some studies show that risk of obstetric complications increases in untreated subclinical hypothyroid women, which was the case even in women having serum TSH levels between 2.2-5 mIU/L, ${ }^{14,15,19}$ results, however, not consistently seen in many similar studies. ${ }^{16,20}$ The American Thyroid Association (ATA) and the Endocrine Society Practice Guidelines have endeavored to clarify how to manage subclinical hypothyroidism and screening for thyroid disorders during pregnancy. Many of these recommendations, though based on expert opinion, clearly indicate gaps in knowledge regarding these important issues that deserve special attention. This review highlights the similarities and differences between the ATA and the Endocrine Society Guidelines regarding screening for thyroid disease and treatment of subclinical and overt hypothyroidism while seeking to determine the source of the differences based on current evidence.

\section{METHODS}

Recommendations of the $\mathrm{ATA}^{9}$ and the Endocrine Society Guidelines ${ }^{1}$ were reviewed and compared (pages 1088-1093 and 2544-2550, respectively) for the topics hypothyroidism in pregnancy and thyroid function screening in pregnancy (pages 1107-1111 and 2547-2561, respectively). Similarity between recommendations is designated as "the same" and for the differences more specific comments were added. Tables 1-3 depict similarities or differences between the recommendations of the two sets of guidelines.

\section{RESULTS}

The ATA guidelines included 2 questions and 5 recommendations regarding "screening of thyroid function during pregnancy" and 22 questions and 15 recommendations regarding "hypothyroidism in pregnancy". In the Endocrine Society Clinical practice guidelines, 13 recommendations regarding screening and 8 recommendations regarding the "management of hypothyroidism" were made. Comparisons of the recommendations are summarized in Tables 1-3. For the drawing up of new guidelines, ATA set up a taskforce which consisted of international experts in the field of thyroid disease and pregnancy. The recent ATA guidelines stated that all relevant literature reviews published since 1990 were used as the basis for all of the recommendations proposed. ${ }^{9}$ The update of the Endocrine Society Clinical Practice Guidelines was carried out by members of the taskforce, the members of the Endocrine Society, the Asia and Oceania Thyroid Association and the Latin American Thyroid Society reviewed and commented on it and it was approved by The Endocrine Society Council. 
Table 1. Comparison of recommendations of American Thyroid Association and Endocrine Society on the time and target group for screening for hypothyroidism before and during pregnancy

\begin{tabular}{ll}
\hline \multicolumn{2}{c}{ Who should be screened? } \\
\hline \multicolumn{1}{c}{ American Thyroid Association guideline (2011) } & \multicolumn{1}{c}{ Endocrine Society guideline (2012) } \\
$\begin{array}{ll}\text { There is insufficient evidence to recommend for or against } \\
\text { universal TSH screening at the first trimester visit }\end{array}$ & $\begin{array}{l}\text { Some members recommended screening of all pregnant women } \\
\text { for serum TSH abnormalities by the 9th week or at the time of } \\
\text { their first visit. Others recommended against universal screening } \\
\text { of pregnant women at the time of their first visit and instead } \\
\text { supported aggressive case finding to identify high-risk women }\end{array}$
\end{tabular}

A case-finding approach targeting thyroid function testing

The same

in high-risk groups has been advocated

Who are the high risk group?

Women with a history of thyroid dysfunction and/or

thyroid surgery, Family history of thyroid dysfunction goiter,

Women with anti-thyroid antibodies, having symptoms or

clinical signs suggestive of hypothyroidism. type I diabetes or

autoimmune disorders, history of either miscarriage or preterm

delivery, infertility, prior therapeutic head or neck irradiation,

morbid obesity, age 30 or older, treated with amiodarone or

lithium, a recent (in the past 6 weeks) exposure to iodinated

radiological contrast agents, Residing in an area of known

moderate to severe iodine insufficiency

The same

When should high risk women be screened?

There is insufficient evidence to recommend for or against

TSH testing preconception in women at high risk for hypothyroidism

All pregnant women should be verbally screened at the initial prenatal visit for any history of thyroid dysfunction and/or use of thyroid hormone (LT4) or anti-thyroid medications (MMI, carbimazole, or PTU). Level B-USPSTF

Serum TSH values should be obtained early in pregnancy in women at high risk for overt hypothyroidism

The same
Test high-risk women for elevated TSH concentrations by the ninth week or at the time of their first visit before or during pregnancy

All women considering pregnancy with known thyroid dysfunction and receiving levothyroxine should be tested for abnormal TSH concentrations before pregnancy

\section{DISCUSSION}

An overview of recommendations of the two guidelines shows that they have much in common, and yet both lack strong evidence regarding both screening for thyroid diseases and treatment of subclinical hypothyroidism, the differences being due to lack of evidence to elucidate some of the issues currently unresolved.

Although both guidelines recommend screening of high risk cases and are against mass screening, the justification behind this comment is somewhat weak. The prevalence of thyroid diseases is not low and it is at least similar to those conditions for which generalized screening is recommended. The cost of TSH measurement and treatment, which could prevent serious complications, at least in overt hypothyroidism, is not high. Many people living in iodine insufficient countries could be suffering from overt hypothyroidism and would hence benefit from screening and treatment. The results of the present studies regarding the beneficial effect of screening 
Table 2. Comparison of recommendations of the American Thyroid Association and the Endocrine Society on the management of hypothyroidism before and during pregnancy

Treatment of hypothyroidism (Overt or subclinical) in pregnancy

American Thyroid Association guideline (2011)

Overt hypothyroidism should be treated in pregnancy

Isolated hypothyroxinemia should not be treated in pregnancy

Due to the lack of randomized controlled trials there is insufficient evidence to recommend for or against universal LT4 treatment in TAB negative pregnant women with SCH

Women who are positive for TPOAb and have SCH should be treated with LT4

The recommended treatment of maternal hypothyroidism is with administration of oral LT4.

It is strongly recommended not to use other thyroid preparations such as T3 or desiccated thyroid

The goal of LT4 treatment is to normalize maternal serum TSH values within the trimesterspecific pregnancy reference range (first trimester, $0.1-2.5 \mathrm{mIU} / \mathrm{L}$; second trimester, 0.2-3 mIU/L; third trimester, 0.3-3 mIU/L)

Women with $\mathrm{SCH}$ in pregnancy who are not initially treated should be monitored for progression to $\mathrm{OH}$ with a serum TSH and FT4 approximately every 4 weeks until 16-20 weeks gestation and at least once between 26 and 32 weeks' gestation

Treated hypothyroid patients (receiving LT4) who are newly pregnant should independently increase their dose of LT4 by $\sim 25 \%-30 \%$ upon a missed menstrual cycle or positive home pregnancy test and notify their caregiver promptly. One means of accomplishing this adjustment is to increase LT4 from once daily dosing to a total of nine doses per week $(29 \%$ increase)

There exists great interindividual variability regarding the increased amount of T4 (or LT4) necessary to maintain a normal TSH throughout pregnancy, with some women requiring only $10-20 \%$ increased dosing, while others may require as much as an $80 \%$ increase
Endocrine Society guideline (2012)

The same

No recommendation

T4 replacement in women with $\mathrm{SCH}$ who are TPO-Ab negative subjects

The same

The same

The same

No recommendation

The same

Almost the same

Table 3. Follow-up of pregnant women with overt or subclinical hypothyroidism with LT4 during

\begin{tabular}{lc}
\hline \multicolumn{1}{c}{ Monitoring of pregnant women being treated for hypothyroidism } \\
\hline \multicolumn{1}{c}{ American Thyroid Association guideline (2011) } & Endocrine Society guideline (2012) \\
\hline $\begin{array}{l}\text { In pregnant patients with treated hypothyroidism, maternal serum TSH should be monitored } \\
\text { approximately every } 4 \text { weeks during the first half of pregnancy because further LT4 dose }\end{array}$ & The same \\
adjustments are often required and, maternal TSH should be checked at least once between \\
26 and 32 weeks gestation & The same \\
Following delivery, LT4 should be reduced to the patient's preconception dose. & No recommendation \\
Additional TSH testing should be performed at approximately 6 weeks postpartum & \\
In the care of women with adequately treated Hashimoto's thyroiditis, no other maternal & \\
or fetal thyroid testing is recommended beyond measurement of maternal thyroid function & \\
(such as serial fetal ultrasounds, antenatal testing, and/or umbilical blood sampling) unless & \\
for other pregnancy circumstances &
\end{tabular}

differ. Vaidya et al sought to compare the role of case-finding vs. universal screening for thyroid dysfunction in pregnancy in a randomized controlled trial and demonstrated that screening only high-risk pregnant women failed to detect thyroid dysfunction by up to $69 \%$ during pregnancy. ${ }^{21}$ Negro et al found 
universal screening vs. case-finding did not result in a decrease in adverse pregnancy outcomes. ${ }^{13}$ The ATA guidelines recommend not doing generalized screening, while the Endocrine Society has not reached a conclusion regarding this issue: some of the members agreed to recommend generalized screening, while others have not, which is why two different versions are presented. Hence, due to lack of data, the need for generalized screening remains an ongoing debate.

The question is whether the diagnosis and treatment of subclinical hypothyroidism could result in fewer maternal and fetal complications. Whereas the Endocrine Society guidelines recommend levothyroxine therapy in all women with subclinical hypothyroidism, the ATA guidelines recommend treating women with $\mathrm{SCH}$ who are TPO-Ab positive and women with $\mathrm{SCH}$ with a TSH above $10 \mathrm{mIU} /$ liter, irrespective of thyroid antibody status, due to lack of evidence to show treating SCH with TSH below $10 \mathrm{mIU} /$ liter would be useful. Negro et al found no decrease in pregnancy complications in the screening group but did find treatment of thyroid abnormalities could significantly prevent adverse outcomes in pregnant mothers; their study reported that case-finding strategies failed to detect many pregnancies complicated by thyroid diseases. The results of this study underline the current debate regarding whether all pregnant women should be screened. ${ }^{13}$ Recently, Lazarus et al reported that the IQ levels of offspring born to mildly hypothyroid mothers did not differ from controls and that treatment of hypothyroidism did not improve cognitive function in these children; however, the negative results of their study may be due to the screening that was performed and levothyroxine therapy initiated at up to 20 weeks' gestation, which is too late to have a major influence on brain development. The results of post hoc analyses showed no benefit of screening and treatment in the subgroup of women who had been administered levothyroxine earlier. ${ }^{18}$ The timing of screening could probably interfere in the results of many studies because screening is normally conducted at the time when routine antenatal care is initiated in the majority of women. Also, the time of evaluation of IQ scores of offspring are important. IQ scores were assessed at the age of three by Lazarus et al, an age that may be insensitive to the effects of maternal levothyroxine treatment, ${ }^{18}$ and their results differ from those of another study showing an association between hypothyroidism in women and a lower IQ in offspring for whom psychological testing was performed at 7 years. ${ }^{10}$ In a recent observational study, Ghorbani et al found no difference in IQ level and cognitive performance of children born to LT4-treated hypothyroid mothers, compared to euthyroid subjects. ${ }^{22}$ In contrast, results of other observational studies reported milder neurological deficits in the offspring of hypothyroid mothers. ${ }^{10,23}$ Vaidya et al showed that screening only high-risk pregnant women would result in missing about one third of those with overt/subclinical hypothyroidism. ${ }^{21}$ Wang et al recently showed that a case-finding strategy for screening thyroid function in high-risk Chinese pregnant mothers would miss about $81.6 \%$ of hypothyroid cases. ${ }^{24}$

How to deal with hypothyroxinemia during pregnancy is a matter of debate. Henrichs et al, in a large and diverse population-based study, showed that maternal hypothyroxinemia predicted a higher risk of verbal and nonverbal cognitive delay in early childhood, but TSH levels in early pregnancy across the entire range were not a predictor for cognitive outcomes. ${ }^{25}$ In the Haddow et al study, maternal TSH levels over the 98th percentile accompanied by hypothyroxinemia resulted in neurodevelopmental deficits in offspring. ${ }^{10}$ Another study found that maternal low FT4 level is a major cause leading to poor neurodevelopment of children born to hypothyroxinemic mothers. ${ }^{26}$ The Pop et al study found that children of women with FT4 levels below the 10th percentile, despite having TSH levels within the reference range during the first trimester of pregnancy, are at risk of having a delay in psychomotor development at the age of 2; this however was not the case for those whose mothers had higher levels of fT4 during early pregnancy. ${ }^{17}$

According to current studies, it is impossible to demonstrate causality of the association between the mother's hypothyroxinemia and neurodevelopmental growth of offspring, this being due to wide variations among studies in the definition of hypothyroxinemia, a serious methodological problem. The time for assessment of neurocognitive function also differs between studies as does the definition of the severity of hypothyroxinemia. Well-designed randomized controlled trials are needed to investigate the re- 
lationship between maternal fT4 levels and, more importantly, the effect of treatment during pregnancy and the neurodevelopmental function of offspring. Moreover, long-term follow-up of the cohort is mandatory in terms of psychomotor assessments, because the assessment of these tests in an infant at an early age still has only a limited value in prediction of the child's abilities once he/she has reached school age.

What brings about these different recommendations, especially since the evidence is the same? The differences actually reflect the different levels of evidence used by the two committees and the point of view of each member in making these recommendations. The two guidelines found that many of these observational studies have not yet reached a consensus on recommending a generalized screening program; besides this, at present, no organization recommends universal screening before or during pregnancy. The two guidelines agree on the definition of high-risk pregnant women who need screening to detect thyroid dysfunction.

Despite the rapidly increasing body of evidence, trials addressing the usefulness of treating subclinical hypothyroidism to prevent fetal and maternal complications and the adverse effects on neurocognitive development of offspring are not satisfactory and this issue needs to be definitively resolved in welldesigned RCTs. Though the two guidelines have much in common, the fact that they differ on some vital points results in causing frustration among physicians. Without doubt, one set of unanimously agreed upon guidelines would be of huge benefit. The fact is that the work of clinicians is hampered by both a paucity of high-quality research studies and conflicting data in the field of thyroid and pregnancy, a field which suffers from an insufficient number of randomized controlled trials, of which, to our knowledge, to date just three have been done. We need more well-designed trials to address the many unanswered questions and to elucidate the controversial issues in this field. There are some large-scale, well-designed trials under way including the National Institutes of Health Maternal Fetal Medicine TSH study, the Thyroid AntiBodies and LEvoThyroxine trial (the TABLET trial) and the Subclinical Hypothyroid and Iodine Deficiency in Early Pregnancy and Women Planning for Pregnancy: Screening and Intervention trial (the SHEP study), the results of which will surely cover many important issues in this field.

\section{CONCLUSION}

Overall, though the present data are neither for nor against universal screening at this time, there is unanimity regarding treatment of pregnant women who suffer from overt hypothyroidism. While the results of the current data do not consistently favor treatment of subclinically hypothyroid women, it is clear that the potential benefits outweigh the risks of treating these cases. Meanwhile, a large amount of new and ever-evolving evidence regarding thyroid diseases during pregnancy is being published. Nevertheless, as mentioned above, more RCTs are urgently needed, primarily to confirm the beneficial effects of universal screening as well as of treatment of pregnant women with subclinical hypothyroidism as well as to gather more data that could fill in gaps regarding the important topic of thyroid and pregnancy.

\section{CONFLICT OF INTEREST}

The authors have nothing to declare.

\section{REFERENCES}

1. Glinoer D, 1997 The regulation of thyroid function in pregnancy: pathways of endocrine adaptation from physiology to pathology. Endocr Rev 18: 404-433.

2. Burrow GN, Fisher DA, Larsen PR, 1994 Maternal and fetal thyroid function. N Engl J Med 331: 1072-1078.

3. De Groot L, Abalovich M, Alexander EK, et al, 2012 Management of thyroid dysfunction during pregnancy and postpartum: An endocrine society clinical practice Guideline. J Clin Endocrinol Metab 97: 2543-2565.

4. Quinn FA, Gridasov GN, Vdovenko SA, Krasnova NA, Vodopianova NV, Epiphanova MA, 2005 Schulten M. Prevalence of abnormal thyroid stimulating hormone and thyroid peroxidase antibody-positive results in a population of pregnant women in the Samara region of the Russian Federation. Clin Chem Lab Med 43: 1223-1226.

5. Shields BM, Knight BA, Hill AV, Hattersley AT, Vaidya B, 2013 Five-year follow-up for women with subclinical hypothyroidism in pregnancy. J Clin Endocrinol Metab 98: 1941-1945.

6. Altomare M, LA Vignera S, Asero P, et al, 2013 High prevalence of thyroid dysfunction in pregnant women. J Endocrinol Invest 36: 407-411.

7. Mosso ML, Martínez GA, Rojas MP, et al, 2012 Fre- 
quency of subclinical thyroid problems among women during the first trimester of pregnancy. Rev Med Chil 140: 1401-1408.

8. Soldin OP, Soldin D, Sastoque M, 2007 Gestationspecific thyroxine and thyroid stimulating hormone levels in the United States and worldwide. Ther Drug Monit 29: 553-559.

9. Stagnaro-Green A, Abalovich M, Alexander E, et al, American Thyroid Association Taskforce on Thyroid Disease during Pregnancy and Postpartum 2011 Guidelines of the American Thyroid Association for the diagnosis and management of thyroid disease during pregnancy and postpartum. Thyroid 21: 1081-1125.

10. Haddow JE, Palomaki GE, Allan WC, et al, 1999 Maternal thyroid deficiency during pregnancy and subsequent neuropsychological development of the child. N Engl J Med 341: 549-555.

11. Abalovich M, Gutierrez S, Alcaraz G, Maccallini G, Garcia A, Levalle O, 2002 Overt and subclinical hypothyroidism complicating pregnancy. Thyroid 12: 63-68.

12. Leung AS, Millar LK, Koonings PP, Montoro M, Mestman JH, 1993 Perinatal outcome in hypothyroid pregnancies. Obstet Gynecol 81: 349-353.

13. Negro R, Schwartz A, Gismondi R, Tinelli A, Mangieri T, Stagnaro-Green A, 2010 Universal screening versus casefinding for detection and treatment of thyroid hormonal dysfunction during pregnancy. J Clin Endocrinol Metab 95: 1699-1707.

14. Negro R, Schwartz A, Gismondi R, Tinelli A, Mangieri T, Stagnaro-Green A, 2010 Increased pregnancy loss rate in thyroid antibody negative women with TSH levels between 2.5 and 5.0 in the first trimester of pregnancy. J Clin Endocrinol Metab 95: E44-48.

15. Casey BM, Dashe JS, Wells CE, et al, 2005 Subclinical hypothyroidism and pregnancy outcomes. Obstet Gynecol 105: 239-245.

16. Cleary-Goldman J, Malone FD, Lambert-Messerlian G, et al, 2008 Maternal thyroid hypofunction and pregnancy outcome. Obstet Gynecol 112: 85-92.

17. Pop VJ, Brouwers EP, Vader HL, Vulsma T, van Baar
AL, de Vijlder JJ, 2003 Maternal hypothyroxinaemia during early pregnancy and subsequent child development: a 3-year follow-up study. Clin Endocrinol (Oxf) 59: 282-288.

18. Lazarus JH, Bestwick JP, Channon S, et al, 2012 Antenatal thyroid screening and childhood cognitive function. N Engl J Med 366: 493-501.

19. Benhadi N, Wiersinga WM, Reitsma JB, Vrijkotte TG, Bonsel GJ, 2009 Higher maternal TSH levels in pregnancy are associated with increased risk for miscarriage, fetal or neonatal death. Eur J Endocrinol 160: 985-991.

20. Mannisto T, Vaarasmaki M, Pouta A, et al, 2010 Thyroid dysfunction and autoantibodies during pregnancy as predictive factors of pregnancy complications and maternal morbidity in later life. J Clin Endocrinol Metab 95: 1084-1094.

21. Vaidya B, Anthony S, Bilous M, et al, 2007 Detection of thyroid dysfunction in early pregnancy: Universal screening or targeted high-risk case finding? J Clin Endocrinol Metab 92: 203-207.

22. Behrooz HG, Tohidi M, Mehrabi Y, Behrooz EG, Tehranidoost M, Azizi F, 2011 Subclinical hypothyroidism in pregnancy: intellectual development of offspring. Thyroid 21: 1143-1147.

23. Man EB, Brown JF, Serunian SA, 1991 maternal hypothyroxinemia: psychoneurological deficits of progeny. Ann Clin Lab Sci 21: 227-239.

24. Wang S, Teng WP, Li JX, Wang WW, Shan ZY, 2012 Effects of maternal subclinical hypothyroidism on obstetrical outcomes during early pregnancy. J Endocrinol Invest 35: 322-325.

25-Henrichs J, Bongers-Schokking JJ, Schenk JJ, et al, 2010 Maternal thyroid function during early pregnancy and cognitive functioning in early childhood: the generation R study. J Clin Endocrinol Metab 95: 4227-4234.

26. Morreale de Escobar G, Obregón MJ, Escobar del Rey F, 2000 Is neuropsychological development related to maternal hypothyroidism or to maternal hypothyroxinemia? J Clin Endocrinol Metab 85: 3975-3987. 